\title{
CIENCIA, TÉCNICA Y ALGORITMOS: NOTAS EN TORNO A SU $A$ PRIORI IDEOLÓGICO-CAPITALISTA
}

\section{SCIENCE, TECHNIQUE AND ALGORITHMS: NOTES ABOUT THEIR IDEOLOGICAL-CAPITALIST A PRIORI}

\author{
JORGE LUIS QUINTANA MONTES \\ Corporación Universitaria Rafael Núñez \\ jorge.quintana@curnvirtual.edu.co
}

RECIBIDO: $13 / 07 / 2021$

ACEPTADO: 06/10/2021

Resumen: El objetivo del artículo es ofrecer una aproximación crítica a los algoritmos de aprendizaje automático, entendidos como una nueva faceta del dominio ideológico propio del modo de producción capitalista. En este sentido, el artículo se encuentra constituido por tres momentos temáticos: $i$ ) definición de algoritmos y de machine learning, ii) el asedio comercial como reproducción de la lógica de consumo del capital y iii) microtargeting político, amparado en la desinformación reinante en la esfera pública en tiempos de campaña electoral.

Palabras clave: algoritmos; machine learning; capitalismo; microtargeting; consumo

Abstract: The objective of the article is to offer a critical approach to machine learning algorithms, understood as a new facet of the ideological domain of the capitalist mode of production. In this sense, the article is made up of three thematic moments: $i$ ) definition of algorithms and machine learning, ii) commercial siege as a reproduction of capital's consumption logic and iii) political microtargeting, expressed in the prevailing misinformation in the public sphere in times of electoral campaign.

Keywords: algorithms; machine learning; capitalism; microtargeting; consumption

En el marco de su reflexión tardía, Martin Heidegger destacó como esencia de la técnica moderna lo que denominó Ge-stell. Ésta es entendida por el fenomenólogo como un modo específico de coligar al ente y traerlo al aparecer como stock. Esta lectura 
heideggeriana es apropiada por Marcuse en One-Dimensional Man para pensar el carácter político de la técnica desde su esenciar, no sólo como un dominio del hombre sobre la naturaleza, sino como un dominio del hombre sobre el hombre. El modo de producción capitalista se despliega históricamente a partir de dicha lógica de dominación y explotación, aunque adquiere en su faceta más contemporánea una radical faz científico-técnica.

El objetivo que dirige la realización de esta contribución es pensar los elementos más actuales del carácter científico-técnico del capitalismo, esbozando la forma en que el uso de los algoritmos de machine learning se manifiesta en una doble vía de dominio político: por un lado, reproducen la lógica de consumo capitalista, cristalizando con ello la categoría marcuseana de falsas necesidades. Por otra parte, permiten la manipulación de las redes sociales, haciéndolas un campo de desinformación durante las campañas políticas.

El orden de la exposición será el siguiente: $i$ ) caracterización general de los algoritmos y el machine learning, ii) microtargeting de consumo y iii) microtargeting político.

\section{Algoritmos}

En un sentido amplio, los algoritmos pueden ser entendidos como el conjunto de pasos específicos que permiten la resolución de un problema concreto y delimitado (House of Commons, 2018, p. 7). Esta definición de carácter amplio se restringe a su uso exclusivo en el campo de la informática, cuando se piensan como la resolución de los problemas que ejecuta un aparato, al seguir el conjunto dado de instrucciones: "De acuerdo con las definiciones estándar de ciencias de la computación, un algoritmo es un conjunto de instrucciones para resolver un problema o completar 
una tarea siguiendo un orden secuencial cuidadosamente planeado" (Bucher, 2018, p. 20). Las instrucciones que recibe el algoritmo para la resolución de problemas hacen parte de su input, aunque no se encuentra -dicho input- constituido exclusivamente por ellas. Los datos también son un elemento constituyente del input (Kitchin, 2017, p. 6).

Los algoritmos computacionales procesan, en este orden de ideas, bien instrucciones o bien datos; por tanto, pueden ser entendidos -además de como el conjunto de instrucciones- como el conjunto de operaciones que se realizan sobre los datos. La pregunta que debemos plantear entonces es la siguiente: ¿cuál es el sentido de seguir un grupo de instrucciones dadas y de procesar de datos? La respuesta se encuentra en el control y anticipación: "los algoritmos son usados esencialmente para controlar el flujo de las acciones y los eventos futuros" (Bucher, 2018, p. 28).

La estructura básica de un algoritmo es la del condicional "Si... entonces...": "Si es el caso que A, B y C, entonces hacemos D, E y F". Esto es válido para todo caso en que el antecedente sea verdadero; es decir, que la situación se dé tal cual se espera. En las circunstancias en que no sea así, la estructura básica funciona del siguiente modo: "Si... entonces... si no es el caso... entonces": "Si es el caso que A, B y C, entonces hacemos D, E y F; si no es el caso que A, B y C, entonces hacemos G, H e I". Los algoritmos establecen qué debe pasar y cuándo debe pasar cada momento del procesamiento de datos; esto, a través de un lenguaje de programación que establece el "flujo de control" (el qué y el cuándo) (Bucher, 2018, p. 22).

Esta estructura básica de funcionamiento de los algoritmos, en que se traza con especificidad cada paso del procesamiento en la estructura "Si... entonces", no es el único modo en que ellos funcionan. Existe otro tipo de algoritmos capaces de "aprender" en el proceso, a través de la interacción con los datos, con los 
humanos y con otros algoritmos. Así, en contraste con los algoritmos que arrojan siempre los mismos resultados, hay unos capaces de sufrir modificaciones (Bucher, 2018, p. 24). Estos algoritmos son los de aprendizaje automático (machine learning). A diferencia del rasgo "Si... entonces" de un algoritmo genérico, los algoritmos vinculados con los procesos de machine learning aprenden las instrucciones para la resolución de determinados problemas -o para el procesamiento de datos-, a partir de una casuística que contiene la regla de forma implícita. Esto implica, necesariamente, un entrenamiento del algoritmo. Así, el algoritmo de aprendizaje automático se piensa desde la anticipación de resultados futuros, basándose en casos pasados similares.

Para el caso que aquí interesa nos compete de forma esencial el machine lerning. Este tipo de algoritmos atraviesa de manera silente nuestra vida diaria. Política, guerra, consumo, seguridad, economía, afectividad, etc., todo ello se encuentra determinado en distintos grados por su acción y por la especificidad de su evolución en distintos campos (Roberge \& Seyfert, 2016, p. 1). De acuerdo a lo que indica Adrian Lobe (2019), se han implementado softwares algorítmicos para predecir crímenes, la poca higiene de los restaurantes y el abuso infantil.

Hecha esta indicación es necesario cuestionarnos lo siguiente: ¿en qué sentido son funcionales al sistema capitalista los algoritmos de aprendizaje automático? Para responder este interrogante es preciso señalar que el machine learning es impensable sin su relación con los datos (House of Commons, 2018 , p. 16), por ello el control de los datos es tan importante hoy en día (no hay que olvidar la no tan lejana polémica por la política de manejo y uso de datos por parte de Whatsapp). En virtud de ello, pongamos sobre la mesa una nueva inquietud: ¿qué debemos entender aquí por datos? Datos es el conjunto de información que permite la identificación de un individuo cualquiera (Boldyreva, 
Grishina \& Duisembina, 2018, p. 92). Los datos son esenciales a los algoritmos, pues es desde ellos que se realizan los perfilamientos que permiten la anticipación de las acciones humanas. El perfilamiento debe ser entendido como el procesamiento informático de datos personales que permite predecir y los establecer gustos, preferencias, intereses, movimientos, condición económica, etc., de un sujeto determinado (Bakir, 2020, p. 2).

Una vez se desarrolla el perfil de los usuarios de la web, el siguiente paso es hacer de los algoritmos de machine learning los mediadores entre el individuo y la información que abunda en la red. Establecidos los intereses del individuo, se lo pone de frente con aquellas cosas que prefiere: "Entendido como las instrucciones codificadas que una computadora necesita seguir para realizar una tarea determinada, los algoritmos se implementan para tomar decisiones, ordenar y hacer visible de manera significativa la gran cantidad de datos producidos y disponibles en la Web" (Bucher, 2018, pp. 2-3). Desde aquí surge el doble modo de despliegue del microtargeting: uno de consumo y uno político, y es en este lugar que se constituye el carácter ideológico de los algoritmos. Valga indicar que se entiende por microtargeting la forma en que se desarrolla -a través de herramientas informáticas- publicidades personalizadas que respondan a los intereses concretos de grupos o individuos específicos.

\section{Machine learning y consumo}

De acuerdo a lo indicado por Woolley \& Howard (2016), para 2014 se calculó un aproximado de 10 billones de dispositivos móviles en el mundo, entre Laptops, Tablets y celulares (p. 4884). Esta proliferación de instrumentos técnicos -que viene acompañada 
necesariamente del ensanchamiento en el alcance de la internet, pues la funcionalidad plena de estos aparatos es inconcebible sin conectividad - se ha tornado en el modo más eficiente de sostenimiento del sistema y, por tanto, de dominio del hombre sobre el hombre: cargamos en nuestros bolsillos, morrales y carteras las cadenas y grilletes que nos hacen objeto -casi que gozosamente- del sometimiento.

A juicio de Snake-Beings (2013) las sociedades contemporáneas han sufrido un cambio esencial: muy a pesar de que se edifican a partir de ejercicios de dominio, no lo hacen a través de elementos ideológicos. Lo que llama "sociedades de control", se caracterizan por el monopolio no institucionalizado de la información. El control experimentado es, a su juicio, un control no ideológico sino algorítmico: "Como tal, [los algoritmos] no son intrínsecamente liberadores, sino que constituyen una poderosa herramienta de opresión intelectual que, en mi opinión, reemplaza la opresión ideológica de las tecnologías más antiguas" (Snake-Beings, 2013, p. 1). Aunque no lo comente explícitamente, Snake-Beings parece hacer coincidir de forma exclusiva el concepto de ideología con su rostro institucionalizado.

Seguimos a Snake-Beings cuando indica que el uso de los algoritmos tiene como uno de sus rasgos propios el control social, pero nos distanciamos para afirmar -siguiendo a Mager (2014)que la crítica de las instituciones tradicionales no anula el carácter ideológico de la sociedad; antes bien, ha adquirido una nueva faz que se expresa ahora en elementos como las redes sociales (Facebook, Instagram, Twitter) y los motores de búsqueda (Google). Estos, y en general la web, no pueden ser desgajados del específico horizonte histórico del cual nacen: "He sostenido que los motores de búsqueda no deben verse como externos a la sociedad, sino más bien como negociados y moldeados en la sociedad. Nos muestran el rostro del capitalismo porque nacieron y se criaron en 
una sociedad capitalista. Encarnan una 'ideología algorítmica"” (Mager, 2014, p. 28).

A este respecto, vale indicar que Google desarrolló una tecnología que permitía vincular determinados productos y mercancías con las palabras claves buscadas por sus usuarios. De forma paralela, ejecutó un modelo de negocios que le permitía cobrar a los anunciantes por aparecer en el tope de las opciones (Mager, 2014, p. 29). Con ello se pone en evidencia que el filtro algorítmico de la información responde a un interés económico expreso. Con esto se genera de forma concomitante un primer modo de comprensión del usuario de la web, que lo reduce a simple consumidor de mercancías: "solo vemos lo que dictan nuestras historias de búsqueda anteriores; nuestros historiales de búsqueda están categorizados para conectarse con los intereses comerciales disponibles" (Snake-Beings, 2013, p. 3).

Es justamente porque se comprende al usuario de este modo que el microtargeting adquiere su razón de ser: si los que navegan en la web son consumidores potenciales, y si hay una necesidad del capitalismo de ensanchar los mercados y generar falsas necesidades para reproducirse a través del consumo -como bien expone Marcuse en One-Dimensional Man-, entonces la labor de los algoritmos no es únicamente filtrar la información para nosotros, es exponernos - gracias al perfilamiento- a un conjunto de productos que el sistema intuye que consumiremos, pues ya han sido consumidos por otros que tienen el mismo perfil.

En último término, lo que se genera en la navegación web es lo que Roberge \& Seyfert (2016) denominan un "loop de consumo": un movimiento cíclico y repetitivo, una suerte de bucle fundamental para la lógica económica de la sociedad capitalista: desde el perfilamiento generado a través del procesamiento de datos se realiza una recomendación acertada que deviene en consumo del producto por parte del navegante de la web. Este 
consumo de las recomendaciones produce nuevos datos que permiten hacer recomendaciones futuras cada vez más acertadas y refinadas, dirigiendo y formando con cada nueva recomendación el machine learning- las preferencias y gustos del individuo. Esto implica necesariamente un mayor consumo de los productos ofrecidos, pues coinciden dichas "recomendaciones en bucle" con los gustos y preferencias generados -a su vez- por el mismo sistema. En este caso, el nuevo producto consumido gracias a la sugerencia algorítmica no está en muchos casos siquiera contemplado por parte del individuo; sin embargo, al verlo allí, destacándose, los algoritmos nos invitan y conducen sutilmente a hacerlo nuestro.

Esta capacidad que tienen los algoritmos para reproducir la lógica de consumo del capitalismo contemporáneo expresa justamente su condicionamiento ideológico. Así, y a pesar de la indicación de Snake-Beings, coincidimos con la comprensión del fenómeno que nos ofrece Mager: no sólo es el carácter mercantil que tiene el mayor motor de búsqueda del mundo (Google) lo que ayuda a reproducir el statu-quo. Lo es también el modo en que el consumo se vuelve una meta en sí misma: no consumimos para satisfacer una necesidad real, sino porque el sistema crea una necesidad falsa - por pensarlo con Marcuse- que no tiene una meta distinta que la afirmación de la totalidad del sistema capitalista (Mager, 2014, pp. 33-35).

\section{Microtargeting político}

Nuestro punto de partida es, como en el acápite anterior, el vínculo indisoluble que se teje entre la vida cotidiana, los aparatos y la conectividad a Internet. En este orden de ideas, del mismo modo en que nos encontramos expuestos de un modo constante mientras 
navegamos a la venta de mercancías, asimismo las plataformas digitales se han convertido en un nicho de trabajo político.

El caso del hoy cuestionado presidente de Colombia es un fiel testimonio de esta importancia: una inversión aproximada de un millón de dólares para posicionar positivamente a Iván Duque en redes sociales. Contrato firmado, no como dato menor -de acuerdo a lo indicado en Semana (2020)- en tiempos de pandemia (30 de Abril de 2020), y con dineros destinados para el proceso de paz con la guerrilla de las FARC.

Otro dato no menor que debemos tener en cuenta, también para el caso colombiano -en el marco del paro nacional iniciado el 28 de Abril de 2021-, es la falla sistemática en las telecomunicaciones en Cali, ciudad epicentro de los conflictos en Colombia. Las zonas neurálgicas de la lucha en dicha ciudad han visto afectada su conectividad a internet, así como el servicio telefónico, impidiendo con ello la difusión de las evidencias de los ejercicios de represión, los asesinatos y las violaciones sexuales ejecutadas por miembros de la Policía y el Ejército, bajo el amparo del gobierno nacional. Pese a ello, a más de un mes del inicio de la lucha, Colombia aún resiste. De acuerdo a lo indicado por Hernández (2021), el caso de censura y bloqueo de información en el marco de las protestas en Colombia no hace más que sumarse a otros casos mundiales, a saber: las revueltas en Bangladesh y las elecciones presidenciales en El Congo. La ciencia y la técnica moderna tienen como su $a$ priori, bien lo dijo Marcuse, el dominio del hombre por el hombre, más allá del sistema económico-político (capitalismo o comunismo) existente.

Para Conover, Goncalves, Ratkiewicz, Flammini \& Menczer (2011) las campañas políticas se están desplazando desde medios más tradicionales hacia herramientas de bajo costo con gran alcance. Ello permite una interacción más próxima a los votantes y asegura un intercambio fluido con la base política (Conover et. al., 
2011, p. 192). Internet es -a su juicio- este trampolín del que se sirve la política para impulsarse. Esto explica en gran medida la manera en que el hoy expresidente Donald Trump distribuyó los fondos publicitarios de la campaña presidencial que lo llevaría a la Casa Blanca entre 2017 y 2021. En contraste con Hilary Clinton, que invirtió US\$200.000.000 en publicidad ordinaria $\mathrm{y}$ US\$30.000.000 en la web, Trump invirtió alrededor de US\$90.000.000 en publicidad online (Ortega, 2016). Trump se sirvió de las plataformas digitales para llegar a la ciudadanía en los estados políticamente neurálgicos ¿La razón? El carácter político de los avances científico-técnicos: "Según el Pew Internet and American Life Project, el 73\% de los usuarios adultos de Internet se conectaron en línea para obtener noticias o información sobre política en 2010, y más de uno de cada cinco adultos (22\%) utilizó Twitter o sitios de redes sociales con fines políticos" (Conover et al., 2011, p. 192).

Bakir nos ofrece un interesante listado de la utilidad ofrecida por la base científico-técnica, aplicada a las campañas políticas:

1. El autor indica que Kosinski, Stillwell \& Graepel (2013) desarrollaron un algoritmo que, basado únicamente en los likes que se dan a páginas de Facebook, permite perfilar al individuo dentro de cinco categorías centrales (OCEAN): "Openness to experiences, Conscientiousness, Extroversion, Agreeableness, and Neuroticism" (Bakir, 2020, p. 3). Este mismo algoritmo, además, ayuda a identificar la orientación política, religiosa y sexual de los usuarios de la red social.

2. El perfilamiento ayuda, además, a realizar un microtargeting político, pues permite encontrar votantes específicos que, aunque no se adhieran de forma explícita a una postura política, compartan -o al menos por lo que el perfilamiento basado en datos indica- determinados intereses con aquellos sujetos que sí apoyan abiertamente 
una causa concreta. Así, si $A$ es votante de $T$ y tiene los rasgos $x, y, z$; y $B$ no se afirma como votante de $T$ pero comparte con $A$ los rasgos $x, y, z$, entonces $B$ se vuelve un objetivo concreto de asedio y publicidad política por parte de la campaña de $T$. Como bien indicaron Conover et. al. respecto del perfilamiento en Twitter, una vez se identifican quiénes sí y quienes no asumen una posición política explícita en redes, el trabajo que sigue es ganar votantes en esos que pueden ser persuadidos dada su condición ambigua.

3. Usar las herramientas informáticas en la web para desvirtuar una contienda electoral también es una estrategia común: se utilizan bots para apoyar de forma falsa y aumentar la popularidad de un candidato. Se utilizan Fake News para desprestigiar competidores, o cuentas falsas para difundir mensajes inflamatorios personalizados a través de las redes sociales. Este último fue el caso de las elecciones que ganaría Trump en 2016 (Bakir, 2020, p. 4).

El caso de Cambridge Analytica es crucial -a nuestro juicio- pues hace las veces de testimonio histórico que pone de relieve el carácter político de dominio que late de fondo en la ciencia y la técnica modernas, en este caso, expresado en el uso de información para la manipulación de los electores alrededor del mundo. Cambridge Analytica se presentó como una empresa que, a través del uso de los datos, podía realizar campañas políticas persuasivas (Boldyreva, Grishina \& Duisembina, 2018, p. 95).

El punto de partida para el trabajo de Cambridge Analytica fue la obtención de datos ¿Cómo lo hizo? Gracias a una aplicación que hurtaba no sólo los de sus usuarios directos, sino que a través de estos, los extraía de sus contactos en Facebook. En 2018 se reveló el número de personas directa o indirectamente asociadas a la aplicación: "70.632.350 en EEUU, 1.175 .870 en Filipinas, 
1.096.666 en Indonesia, 1.079.031 en el Reino Unido, 789.880 en México, 622.161 Canadá, 562.455 en India, 443.117 en Brasil, 427.446 en Vietnam y 311.127 en Australia" (Bakir, 2020, p. 7). La información se extrajo de forma engañosa, pues se indicó que la app no obtendría los datos debido a su uso. Con los datos obtenidos se perfiló algorítmicamente a los usuarios de la red social. Se contrastó este perfil con los datos de los votantes estadounidenses y se empezó una campaña publicitaria personalizada en función de los resultados.

Una vez perfilados psicográficamente los usuarios de la red, se procedía a lo que llama Bakir "operaciones psíquicas". Con este concepto alude a la manipulación política a que se someten los electores a través de una exposición a información engañosa $o$ parcial. La exposición engañosa tiene lugar a través de un microtargeting político en las redes sociales y la web en general. Allí el individuo se encuentra sometido a un asedio meticuloso de información prescrita con el objetivo de que actúe de una manera deseada: comportándose políticamente de un modo pre-establecido. Durante la revisión legal del procedimiento de Cambridge Analytica, se indicó que se usaron los datos obtenidos engañosamente para manipular los votantes. Con un perfil general de personalidad que bosquejaba los rasgos propios de comportamientos paranoicos e intolerantes, se pudo identificar usuarios que respondían al perfil y hacerlos objeto de un asedio informativo, con la intención de que sus acciones subsiguientes fuesen convenientes a los intereses de quien generó el asedio.

\section{A modo de conclusión}

Argumentos de Razón Técnica, nº 24, 2021, pp. 178-193 
A lo largo de esta breve contribución, intentamos seguir el impulso marcuseano para esbozar el modo en que la ciencia y la técnica modernas se encuentran al servicio del statu-quo capitalista, en lo que al orden económico y político se refiere. En nuestro habitar contemporáneo, lo fundamental es el modo en que los algoritmos de aprendizaje automático son funcionales para determinar nuestra comprensión del mundo: generan gustos que, una vez generados, son perpetuados y nos reconducen hacia una lógica perpetua de consumo. El loop de los algoritmos es un loop de consumo.

Asimismo, y en lo que a las campañas políticas se refieren, el machine learning permite el perfilamiento y asedio político, sirviéndose de las redes sociales, para desvirtuar a través de la propaganda informativa lo que acontece. Las Fake News se transforman en una herramienta crucial para generar una ventana de visibilidad miope de la vida pública. Ahora bien, vale indicar lo siguiente: el triunfo político de Trump sobre Clinton a través del uso de elementos técnicos no implica que, en un caso hipotéticamente contrario; es decir, bajo una supuesta victoria de Clinton, entonces la ciencia y la técnica moderna hubiesen estado al servicio de un proyecto emancipatorio. Bajo nuestro análisis del problema subyace una premisa marcuseana fundamental, a saber: el cierre del universo político. No hay una oposición concreta, efectiva, real al statu quo. Lo que encontramos son alternativas que se mueven dentro de la misma lógica del sistema. El triunfo del hoy presidente Joe Biden, aunque pueda dar muestras de una mesura política, no supone una disolución de la naturaleza misma del capitalismo contemporáneo. No hay allí, en lo absoluto, un cambio cualitativo. La ciencia y la técnica modernas no hacen más que ofrecer, en un universo político cerrado, una versión menos radical -al menos en apariencia- de la lógica del capital. En el reino de la pura positividad, lo único que tiene lugar es una alternativa, una 
versión rebajada de lo mismo: así como nos sentimos libres eligiendo el color de nuestro nuevo celular comprado por internet, del mismo modo nos sentimos libres eligiendo entre dos opciones presidenciales que no pretenden subvertir el statu quo. A lo sumo, pueden aspirar a hacerlo más tolerante $\mathrm{y}$, por tanto, integrador de la negatividad.

\section{Referencias}

Bakir, V. (2020). Psychological Operations in Digital Political Campaigns: Assessing Cambridge Analytica's Psychographic Profiling and Targeting. Frontiers in Communication, 5(67). https://doi.org/10.3389/fcomm.2020.00067

Boldyreva, E.; Grishina, N. \& Duisembina, Y. (2018). Cambridge Analytica: Ethics and Online Manipulation with Decision-Making Process. The European Proceedings of Social \& Behavioural Sciences (18th PCSF 2018 Professional Culture of the Specialist of the Future) 51, 91-102. Future Academy. https://dx.doi.org/10.15405/epsbs.2018.12.02.10

Bucher, T. (2018). If ... then. Algorithmic Power and Politics. Oxford University Press.

Conover, M.; Goncalves, B.; Ratkiewicz, J.; Flammini, J. \& Menczer, F. (2011). Predicting the Political Alignment of Twitter Users. 2011 IEEE Third International Conference on Privacy, Security, Risk and Trust and 2011 IEEE Third International Conference on Social Computing, 192-199. https://doi.org/10.1109/PASSAT/SocialCom.2011.34 Hernández, J. (2021, 6 de Mayo). La interrupción de internet durante las protestas enardece a los manifestantes en Colombia. El País. https://elpais.com/internacional/2021-05-06/la-interrupcion- 
de-internet-durante-las-protestas-agita-a-los-manifestantes-encolombia.html

Heidegger, M. (2000). Vorträge und Aufsätze (GA 7). Vittorio Klostermann.

Heidegger, M. (2009). Zur Metaphysik-Neuzeitlichen WissenschaftTechnik (GA 76). Vittorio Klostermann.

House of Commons (Science and Technology Committee) (2018). Algorithms in decision-making. Fourth Report of Session 2017-19. https://www.regulation.org.uk/library/2018-

response_to_algorithms_report.pdf

Kitchin, R. (2017). Thinking critically about and researching algorithms. Information, Communication \& Society, 20(1), 14-29.

Kosinski, M.; Stillwell, D. \& Graepel, T. (2013). Private traits and attributes are predictable from digital records of human behavior. Proceedings of the National Academy of Sciences of the United States of America, 110, 5802-5805. https://doi.org/10.1073/pnas.1218772110

Mager, A. (2014). Defining Algorithmic Ideology: Using Ideology Critique to Scrutinize Corporate Search Engines. TripleC, 12(1), 28-39.

Marcuse, H. (2002). One-Dimensional Man. Routledge.

Ortega, A. (2016, 22 de Noviembre). Social media and democracy: Trump harnessed the power of algorithms. Real Instituto Elcano [Royal Institute]. https://blog.realinstitutoelcano.org/en/socialmedia-democracy-trump-harnessed-power-algorithms/

Roberge, J. \& Seyfert, R. (2016) What are algorithmic cultures? En R. Seyfert \& J. Roberge (Eds.), Algorithmic Cultures. Essays on meaning, performance and new technologies (pp. 1-25). Routledge. Semana (2020, 4 de Mayo). ¿Se usaron dineros de la paz para posicionar imagen de Duque en redes? https://www.semana.com/semana-tv/semana- 
noticias/articulo/dineros-para-la-paz-fueron-para-posicionarimagen-del-presidente-duque/669003/

Snake-Beings, E. (2013). From Ideology to Algorithm: the Opaque Politics of the Internet. Transformations, 23, 1-8.

Woolley, S. \& Howard, P. (2016). Political Communication, Computational Propaganda, and Autonomous Agents. International Journal of Comunications, 10, 4882-4890. 